\title{
A STUDY ON THE ASSESSMENT OF SAFETY AND EFFICACY OF INTRAMUSCULAR PARECOXIB IN ADULT PATIENTS FOR POSTOPERATIVE ANALGESIA
}

\author{
Ramesh M1, Geetanjali S. Masamaddi2, Shamshuzoha M³
}

${ }_{1}^{1}$ Assistant Professor, Department of Emergency Medicine, PESIMSR, Kuppam, Chittoor, Andhra Pradesh.

${ }^{2}$ Assistant Professor, Department of Anaesthesia, SIMS, Shivamogga.

${ }^{3}$ Senior Resident, Department of Anaesthesia, KIMS, Bangalore.

\section{ABSTRACT}

\section{BACKGROUND}

Relieving pain is one of the fundamental responsibilities of medical practitioners and is frequently a primary goal of patients seeking care. Many published reviews have outlined this problem, some showing that up to $70 \%$ of patients receive ineffective, inadequate or delayed pain relief. The pain that accompanies surgical procedures remains prevalent and is an aspect of the perioperative experience that generates the greatest concern for patients about to undergo surgery. There is also a growing concern that acute painful experiences can also lead to long-term painful consequences, even when tissue healing appears to be complete.

The objective of our study is to assess intramuscular Parecoxib with regard to its efficacy in pain relief, duration of action, need for supplements, safety and adverse effects.

\section{MATERIALS AND METHODS}

This study comprised of 100 postoperative patients of both sexes between age group of 18-70 years who were scheduled for various elective surgical procedures and all those who satisfied inclusion and exclusion criteria. All patients were visited preoperatively, a written informed consent was taken and premedicated with diazepam $0.2 \mathrm{mg} / \mathrm{kg}$ body weight at night. Parecoxib sodium $40 \mathrm{mg}$ IM was given in the recovery room, when patient complained of pain and injection time was noted. The time of onset of action of Parecoxib, duration of analgesia, quality of analgesia were recorded by VAS. Heart rate and blood pressure were recorded at intervals. Supplementary doses of Parecoxib necessary was administered and the time noted. Any adverse effects were noted. The observations were recorded, tabulated and subjected to statistical analysis.

\section{RESULTS}

In the study group, 45 were male and 55 were female. Mean duration of analgesia (VAS of 6 and below) was $13 \pm 2.74$ hours. Quality of analgesia was excellent to good (VAS 1-2) in 55\% of patients. There was no significant change in blood pressure or heart rate for 2-8 hours after intramuscular administration. There were $9 \%$ of patients with nausea and vomiting, $4 \%$ with headache and $3 \%$ with pruritus.

\section{CONCLUSION}

Intramuscular Parecoxib is useful for pain relief in postoperative analgesia with half the number of patients in study enjoying excellent analgesia (VAS of 2 and below) at 2 hours. $62 \%$ of the patients had VAS of 6 and below at 12 hours after intramuscular administration. $7 \%$ of the patients in the study needed supplementation (rescue doses) of 40 mg of Parecoxib sodium intramuscularly at 10 hours.

\section{KEYWORDS}

Parecoxib, Intramuscular, Visual Analogue Scale, Pain Intensity Difference, Pain Relief.

HOW TO CITE THIS ARTICLE: Ramesh M, Masamaddi GS, Shamshuzoha M. A study on the assessment of safety and efficacy of intramuscular parecoxib in adult patients for postoperative analgesia. J. Evolution Med. Dent. Sci. 2017;6(20):1585-1588, DOI: $10.14260 /$ Jemds/2017/348

\section{BACKGROUND}

Pain is defined according to the International Association for the Study of Pain, "as an unpleasant sensory or emotional experience associated with actual or potential tissue damage or described in terms of such damage." 1 Effective control of postoperative pain is one of the most important pressing issues in the field of surgery and one that has significant impact on our health care system. ${ }^{1}$

Financial or Other, Competing Interest: None.

Submission 30-01-2017, Peer Review 25-02-2017,

Acceptance 02-03-2017, Published 09-03-2017.

Corresponding Author:

Dr. Geetanjali S. Masamaddi,

H. No. 326, Shubhan, $6^{\text {th }}$ Cross, $3^{\text {rd }}$ Main

Ashwath Nagar, Shivamogga-577201.

E-mail: geetp11@gmail.com

DOI: $10.14260 /$ jemds $/ 2017 / 348$
Postoperative pain control is generally best managed by Anaesthesiologist because they are well versed with regional techniques of Anaesthesia as well as pharmacological expertise in analgesia.

Apart from obvious humanitarian reasons effective postoperative analgesia results in decreased incidence of respiratory and cardiovascular complications, early return of gastrointestinal motility, early ambulation and discharge from hospital.

Most Important factors that Influence the Accuracy, Quality and Duration of Postoperative Pain Include

1. The site, nature and duration of operation, the type of incision and the amount of intra-operative trauma.

2. The physiological and psychological makeup of the patient.

Historically, the treatment of postoperative pain has been 
given a low priority by both surgeon and anaesthesiologists. Anaesthesia based postoperative pain relief services were developed in the late 1980s to address the inadequacy of pain management in major surgical cases.

Effective pain control is an important aspect of optimal care of surgical patients. Despite advances in knowledge of pathophysiology, pharmacology of analgesics and development of more effective techniques for post-operative pain control many patients continue to experience appreciable discomfort. ${ }^{2}$

Newer developments in nerve physiological techniques are providing insights into effect of pharmacologically active agents upon the specific areas of CNS.

In recent years information has been accumulating about specific sites within the CNS that are likely to be involved with modification of physiology and pharmacology of pain.

There are different methods of postoperative pain management like systemic opioids, intraspinal opioids, epidural anaesthesia, non-opioid analgesics, transcutaneous electrical nerve stimulation (TENS), psychological and other methods.

Use of traditional NSAIDs have many side effects like gastritis, peptic ulceration, bleeding from upper GI tract, delayed platelet inhibition, headache, vertigo, insomnia, bronchospasm, allergic reaction, anaemia, nephrotoxicity, salt and water retention. 3,4

Parecoxib is the first selective cox-2 inhibitor for parenteral administration. Parecoxib is 28,000 times more selective to cox- 2 than cox-1. It has faster onset of action, analgesic efficacy superior to intravenous morphine. It does not interfere with platelet function, no dose adjustments required in elderly and in mild hepatic and renal impairment. It is free from adverse effects like respiratory depression. It has superior gastrointestinal tolerability, free from drug dependence and therapeutic tolerance.

\section{MATERIALS AND METHODS}

This study was conducted in the Department of Anaesthesiology, Kempegowda Institute of Medical Sciences and Research Centre, Bangalore from June 2003 to 2005 after obtaining ethical committee clearance.

The study comprised of 100 patients of both sexes in the age group of 18 to 70 years, who are scheduled for various elective surgical procedures and all those who satisfied inclusion and exclusion criteria.

\section{Inclusion Criteria}

1. Males and/or females between 18-70 years.

2. Postoperative patients in the recovery room who have undergone various procedures like gynaecological, orthopaedic, general or dental surgery.

\section{Exclusion Criteria}

1. Pregnant or lactating women.

2. Patients with history of hypersensitivity to NSAIDs.

3. Patients with conditions predisposing to gastrointestinal dysfunction (e.g. history of peptic ulcers, upper gastrointestinal disease, ulcerative colitis, smoking, advanced age, concurrent corticoids, alcohol abuse, etc.).

4. Patients with a history of bleeding tendencies, cirrhosis and oesophageal varices.

5. Patients who may require concomitant therapy with low dose aspirin, Warfarin, antiepileptics, oral hypoglycaemic drugs, fluconazole, ketoconazole, etc.

6. Patients with severe cardiac, hepatic, renal or cerebrovascular disease, malignancy, chronic uncontrolled systemic disease e.g. diabetes, hypertension, asthma, collagen disorders, etc.

\section{Procedure}

All patients were visited preoperatively. Detailed history was taken and general physical examination and systemic examination was done and relevant investigation was done to check whether patients satisfied inclusion and exclusion criteria.

Written informed consent was taken from all the patients who satisfied inclusion and exclusion criteria. Advantages and consequences explained to them in their own language.

\section{Premedication}

All patients were premedicated with oral dose of diazepam $0.2 \mathrm{mg} / \mathrm{kg}$ at night.

\section{Postoperative Analgesia}

After surgical procedure patients were shifted to recovery room and injection Parecoxib sodium $40 \mathrm{mg}$ IM was given at first complaint of pain by the patient, this is taken as time zero. Subsequently, patient is observed for pain relief at 15 min., 30 min., 1 hr., 2 hr., 4 hr., 6 hr., 8 hr., 10 hr., 12 hr. and 24 hours using VAS scale. Pain relief at 0,12 and $24 \mathrm{hr}$. is evaluated as per pain relief scale (poor, good, fair and excellent) and duration of analgesia was calculated from time zero to resurfacing of pain. Rescue medication (second dose of Parecoxib) was administered at VAS 7 or above. Any adverse events or side effects were noted.

Visual analogue score was calculated as follows: No pain was given as " 0 " score, while worst ever felt pain was given "10" score. Scores 1, 2, 3 were considered as mild pain. 4, 5, 6 as moderate pain and 7, 8, 9 as severe pain. The time of onset of action of Parecoxib, duration of analgesia and quality of analgesia were recorded.

\section{The Quality of Analgesia is graded as follows}

- Excellent - No pain, comfortable.

- Good - Report pain on direct question.

- Fair - Pain, low attention of attending doctor.

- Poor - Patient in agony and restless.

Heart rate and blood pressure were recorded at intervals. Adverse effects like nausea, vomiting, abdominal fullness, abdominal pain, pruritus, bleeding were observed. The observations were recorded, tabulated and subjected to statistical analysis.

\section{Statistical Analysis}

The data collected in this study is analysed statistically through SPSS 11.0 by computing descriptive statistics like mean, standard deviation, standard error of mean and $95 \%$ confidence interval for mean. Mean of the statistical inference is obtained by comparing the difference in means through the independent sample student's t test (under the assumptions that the population's variances are not known). The difference is considered statistically significant whenever $\mathrm{p}$ value is 0.05 . 


\section{RESULTS}

In the present study, it was observed that of the 100 subjects studied 45 (45.0\%) were male, $55(55.0 \%)$ were female, and between 21-50 years of age both male and female constituted about $84.0 \%$. Further, of the 45 males, majority i.e., 35 (77.8\%) were in the age group of 21-50 years whereas among the 55 females 49 (89.1\%) were belonging to $21-50$ years.

In this study, the mean weight of male was 56.09 with a SD of 5.116 whereas for female it was 54.45 with a SD of 4.992. The overall mean weight was 55.19 and SD of 5.008. However, between male and female, there was no significant difference.

In this study, the pain intensity measured by visual analogue scale reveals from table 1 that the mean \pm SD at baseline was $3.43 \pm 0.956$, which was reduced to $2.63 \pm 1.031$ at 15 minutes, $1.76 \pm 0.712$ at 30 minutes, $1.71 \pm 0.832$ at 1 hour and $1.66 \pm 0.794$ at 2 hours. But from $4^{\text {th }}$ hour onwards, the pain intensity has increased gradually from $1.81 \pm 0.982$ to $7.35 \pm 0.968$ till 24 hours. Further, it was noticed that the reduction from 0-15 minutes and 15-30 minutes was found to be statistically significant $(\mathrm{p}<0.001)$. However, though there was reduction from 30 minutes- 1 hour ( $p>0.657$ ), 2 hours $(p>0.649)$ and a minimal increase from 2 hours 4 hours ( $p>0.152$ ) which was not statistically significant. (Graph 1).

The average duration of analgesia with good pain relief was about 12-14 hours which was seen in 23 males (51.11\%) and in 27 females (49.09\%). Duration of analgesia was more in males compared to females. However, the difference was not statistically significant (Table 2).

In the present study of the 45 males, 18 (40\%) reported good relief, so also among females 23 (41.8\%). Pain relief was fair among 13 (28.88\%) males and 11 (20\%) females. The excellent pain relief was slightly high among females, i.e. 8 $(14.5 \%)$ as compared to 6 in males $(13.25 \%)$. The poor response to pain relief was also noticed more in females i.e. $13(23.63 \%)$ compared to $8(17.77 \%)$ in males. However, the difference was not found to be statistically significant. (Graph 2).

\begin{tabular}{|c|c|c|c|c|c|c|c|c|c|c|c|}
\hline \multirow{2}{*}{$\begin{array}{c}\text { Study } \\
\text { Group }\end{array}$} & \multicolumn{10}{|c|}{ No. of Patients with Pain } \\
\cline { 2 - 13 } & $\mathbf{0}$ & $\mathbf{1}$ & $\mathbf{2}$ & $\mathbf{3}$ & $\mathbf{4}$ & $\mathbf{5}$ & $\mathbf{6}$ & $\mathbf{7}$ & $\mathbf{8}$ & $\mathbf{9}$ & $\mathbf{1 0}$ \\
\hline $\begin{array}{c}0 \\
\text { min. }\end{array}$ & - & 5 & 6 & 42 & 35 & 12 & - & - & - & - & - \\
\hline 15 min. & - & 9 & 49 & 14 & 26 & 2 & - & - & - & - & - \\
\hline 30 min. & - & 40 & 44 & 16 & - & - & - & - & - & - & - \\
\hline 1 hour & - & 50 & 32 & 15 & 3 & - & - & - & - & - & - \\
\hline 2 hours & - & 51 & 34 & 14 & 1 & - & - & - & - & - & - \\
\hline 4 hours & - & 48 & 32 & 13 & 5 & 2 & - & - & - & - & - \\
\hline 6 hours & - & 32 & 30 & 20 & 12 & 5 & 1 & - & - & - & - \\
\hline 8 hours & - & 26 & 23 & 16 & 27 & 6 & 1 & - & 1 & - & - \\
\hline $\begin{array}{c}10 \\
\text { hours }\end{array}$ & - & 8 & 19 & 13 & 39 & 19 & 2 & - & - & - & - \\
\hline $\begin{array}{c}12 \\
\text { hours }\end{array}$ & - & - & 1 & 6 & 15 & 11 & 62 & 3 & 2 & - & - \\
\hline $\begin{array}{c}24 \\
\text { hours }\end{array}$ & - & - & - & - & 1 & - & 28 & 6 & 64 & 1 & - \\
\hline
\end{tabular}

Table 1. Pain Intensity of Study Group measured by VAS

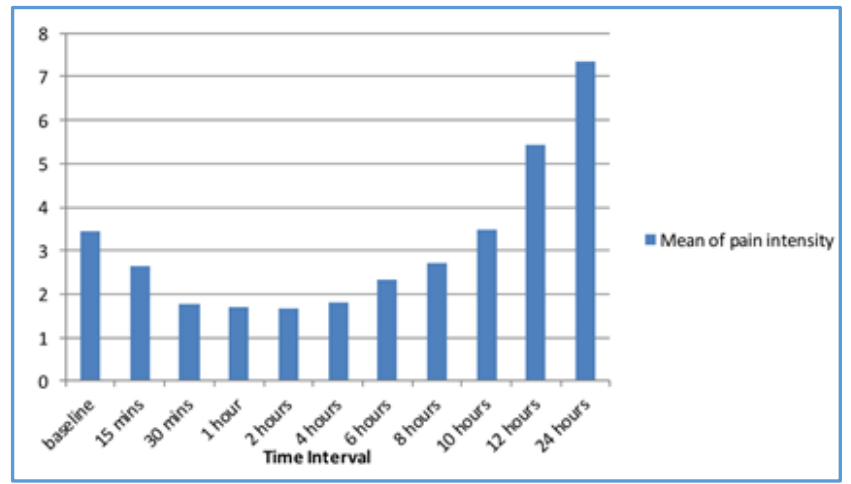

Graph 1. Mean Pain Intensity

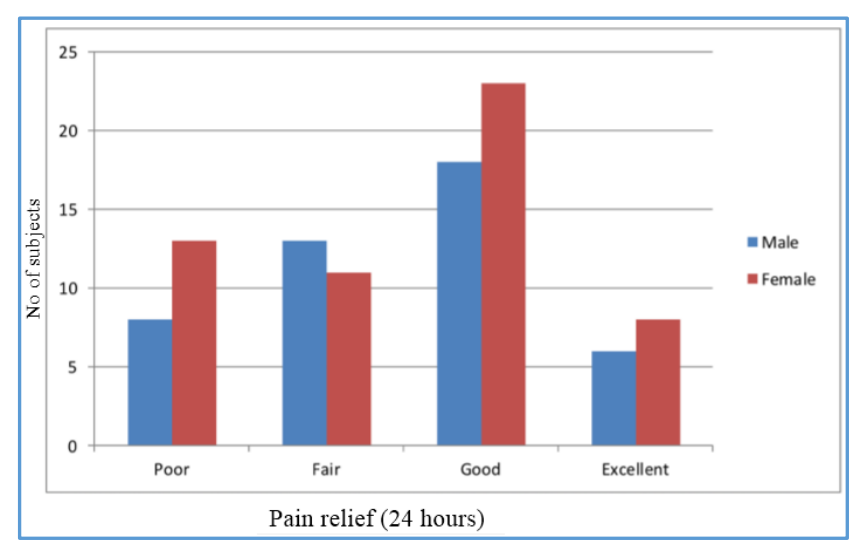

Graph 2. Distribution of Pain Relief in 24 hours

\begin{tabular}{|c|c|c|c|}
\hline \multirow{2}{*}{ Duration of Analgesia } & \multicolumn{2}{|c|}{ Sex } & \multirow{2}{*}{ Total } \\
\hline & Male & Female & \\
\hline $4-6$ & $1(2.2)$ & - & 1 \\
\hline $6-8$ & $2(4.4)$ & $1(1.81)$ & 3 \\
\hline $8-10$ & $1(2.2)$ & $3(5.45)$ & 4 \\
\hline $10-12$ & $4(8.8)$ & $6(10.90)$ & 10 \\
\hline $12-14$ & $23(51.11)$ & $27(49.09)$ & 50 \\
\hline $14-16$ & $9(20.0)$ & $10(1.81)$ & 19 \\
\hline $16-18$ & $4(8.8)$ & $5(9.09)$ & 9 \\
\hline $18-20$ & $1(2.2)$ & $1(1.81)$ & 2 \\
\hline $20-22$ & - & $1(1.81)$ & 1 \\
\hline $22-24$ & - & $1(1.81)$ & 1 \\
\hline Total & 45 & 55 & 100 \\
\hline
\end{tabular}

\section{DISCUSSION}

Management of acute pain is one of the fundamental responsibilities of medical practitioner and is gaining importance. To prevent pain from becoming chronic, agents used for the management of postoperative (surgical) pain relief include NSAIDs, opioids, and local anaesthetics.

Many published reviews have outlined the problem showing that up to $70 \%$ of patients have ineffective, inadequate or delay in pain relief.1,2,4 The pain that accompanies surgical procedures generates the greatest concern for patients about to undergo surgery. There is also growing recognition that acute painful experiences can also lead to long-term painful consequences even when tissue healing appears to be complete. Conventional parenteral NSAIDs have been associated with adverse effects like gastric and peptic ulceration, bleeding from upper gastrointestinal tract, delayed clotting and platelet aggregation, nephrotoxicity, salt and water retention. Conventional 
opioids are associated with side effects like respiratory depression, nausea, vomiting, sedation and constipation.

The novel Cox- 2 inhibitor Parecoxib hydrochloride, the only injectable of Cox-2 selective inhibitor, a prodrug of valdecoxib is being developed for the management of postoperative pain in various surgical procedures.

All the patients in our study received intramuscular Parecoxib only after they complained of pain. Hence, interference by residual effect of intraoperative analgesia or spinal analgesia is ruled out.

P J Desjardins, E Grossman et al $^{5}$ studied analgesic efficacy in healthier adult patients after oral surgery, while comparing the efficacy and tolerability of IV and IM routes of administration. Patients experienced moderate-to-severe pain after extraction of $>2$ impacted third molars randomised to receive Parecoxib sodium $20 \mathrm{mg}$ IM, $20 \mathrm{mg}$ IV, $40 \mathrm{mg}$ IM or $40 \mathrm{mg} \mathrm{IV}$; Ketorolac $60 \mathrm{mg}$ or placebo. Both doses of Parecoxib were comparable to Ketorolac $60 \mathrm{mg} I \mathrm{M}$ in time to onset of analgesia, but Parecoxib had a significantly longer duration of action. They concluded that Parecoxib IV and IM provided effective analgesia.

Karim A, Laurent $\mathrm{A}$ et $\mathrm{al}^{6}$ studied intramuscularly Parecoxib sodium in normal subjects to determine pharmacokinetics, safety, tolerability of single, raising intramuscular doses and single maximum tolerated dose of IM Parecoxib sodium in 56 male volunteers aged 18 to 45 years with doses of Parecoxib sodium $1 \mathrm{mg}, 2 \mathrm{mg}, 10 \mathrm{mg}, 20$ $\mathrm{mg}$ and $40 \mathrm{mg}$ or matching placebo. All single IM doses up to maximum of $40 \mathrm{mg}$ of Parecoxib were well tolerated.

In our study, we used $40 \mathrm{mg}$ Parecoxib sodium intramuscularly, for postoperative pain relief. IM Parecoxib is useful for pain relief with half the number of patients in study, enjoying excellent analgesia (VAS of 2 and below) at 2 hours. $62 \%$ of patients had moderate analgesia (VAS score of 6 and below) at 12 hours after IM administration.

Rasmussen G. L. Steckner K. et $\mathrm{al}^{7}$ studied intravenous Parecoxib sodium for acute pain after orthopaedic knee surgery in 208 healthy patients, Parecoxib $20 \mathrm{mg}$ and $40 \mathrm{mg}$ comparing morphine $4 \mathrm{mg}$ and Ketorolac $30 \mathrm{mg}$. The level and duration of analgesia were significantly superior with Parecoxib sodium than with morphine. Parecoxib sodium was well tolerated in IV doses $40 \mathrm{mg}$, as effective as $30 \mathrm{mg}$ of ketorolac and is more effective than morphine $4 \mathrm{mg}$ and therefore has potential for widespread utility in postoperative management.

In our study, with parecoxib sodium $40 \mathrm{mg}$ intramuscularly, the duration of action was 12 to 13 hours in $51.1 \%$ of the patients. They had excellent-to-good quality of analgesia (VAS 1-2) in $55 \%$ of the patients.

Cheer S. M., Goa K. L. ${ }^{8}$ reported that the most common adverse events irrespective of treatment (Parecoxib,
Ketorolac or placebo) after dental surgery was nausea, alveolar osteitis, dizziness and headache. Nausea, abdominal pain, headache, abdominal fullness, dizziness and back pain, vomiting, tachycardia, somnolence and pruritis occurred in $10 \%$ of Parecoxib recipients after gynaecological surgery.

In our study, with $40 \mathrm{mg}$ of Parecoxib sodium intramuscularly for 100 patients, we encountered nausea and vomiting $9 \%$, pruritus $3 \%$; and headache in $4 \%$ occurred between 3 and 4 hours after the drug administration.

No cardiovascular adverse effects were noted in the given study and all patients remained haemodynamically stable throughout the study period and with better pain relief.

\section{CONCLUSION}

It is concluded that intramuscular Parecoxib is useful for pain relief with half the number of patients in study enjoying excellent analgesia (VAS of 2 and below) at 2 hours. $62 \%$ of the patients had VAS of 6 and below at 12 hours after intramuscular administration. $7 \%$ of the patients in the study needed supplementation (rescue doses) of $40 \mathrm{mg}$ of Parecoxib sodium given intramuscularly at 10 hours.

\section{REFERENCES}

[1] Beneditti C, Bonice JJ. Pathophysiology and therapy of postoperative pain: a review. In: Beneditti C. edr. Advances in pain research and therapy. Vol 7. New York: Raven Press 1984:373-402.

[2] Ready BL, Oden R, Chadwick HS, et al. Development of anaesthesiology - based postoperative pain management service. Anaesthesiology 1998;68(1): 100-6.

[3] Goyal M. Tramadol for postoperative pain relief. Surg 2000;4(11):17-20.

[4] Michael A. Ashburn and brain ready L. Treating acute and chronic pain into $21^{\text {st }}$ century. Advances in anesthesia. Mosby year book Inc., 1996:13.

[5] Desjardins PJ, Grossmann E, Kuss ME, et al. The injectable cyclooxygenase-2 specific inhibitor parecoxib sodium has analgesic efficacy when administered preoperatively. Anesth Analg 2001;93(2):721-7.

[6] Karim A, Lauren A, Slater ME, et al. A pharmacokinetic study of intramuscular (i.m.) parecoxib sodium in normal subjects. J Clin Pharmacol 2001;41(10): 1111-9.

[7] Rasmussen GL, Steckner K, Hogue C, et al. Intravenous parecoxib sodium for acute pain after orthopaedic surgery. Am J Orthop 2002;31(6):336-43.

[8] Cheer SM, Goa KL. Parecoxib is well tolerated after dental, gynaecological or orthopaedic surgery. Drugs 2001;61(8):1133-41. 